\title{
Mirror lightweight for a spaceborne remote sensing instrument
}

\author{
Chia-Yen Chan ${ }^{1}$, Bo-Kai Huang ${ }^{2}$, Ting-Ming Huang ${ }^{1}$, Yi-Cheng Chen ${ }^{2, *}$, and Fong-Zhi Chen ${ }^{1}$ \\ ${ }^{1}$ Instrument Technology Research Center, National Applied Research Laboratories, HsinChu, Taiwan \\ ${ }^{2}$ Department of Mechanical Engineering, National Central University, Taoyuan City, Taiwan
}

\author{
Article history: \\ Received 24 November 2015 \\ Revised 18 March 2016 \\ Accepted 29 March 2016

\section{Keywords:} \\ Mirror lightweight, Taguchi Method, \\ Finite element analysis, Mass reduc- \\ tion ratio, Wavefront aberration, \\ Space specification

\section{Citation:} \\ Chan, C. Y., B. K. Huang, T. M. \\ Huang, Y. C. Chen, and F. Z. \\ Chen, 2017: Mirror lightweight \\ for a spaceborne remote sensing \\ instrument. Terr. Atmos. Ocean. \\ Sci., 28, 139-147, doi: 10.3319/ \\ TAO.2016.03.29.02(EOF5)
}

\begin{abstract}
The paper aims at obtaining the optimum lightweight configuration for a primary mirror (M1) with honeycomb patterns for a space satellite. The finite element analysis and Zernike polynomial fitting based on the Taguchi Method are applied to the whole optimization process. Geometrical control factors and levels were selected to minimize the ratio of various mass reduction ratios (MRRs) to the product of the corresponding maximum mirror structural deflection and optical surface peak-tovalley wavefront aberrations under launch accelerations. The optimum lightweight M1 with a MRR of 0.5 and a mass of $9.72 \mathrm{~kg}$ is attained and a mirror blank based on this design was manufactured. The performance of the optimum lightweight mirror is simulated and the simulation results satisfy the requirements of space specifications.
\end{abstract}

\section{INTRODUCTION}

Mass budget control is an important and essential issue for developing a spaceborne remote sensing instrument (RSI) system. Following the increasing demand for space optics in large size for improving RSI system resolution, the mass elimination of optical mirrors without losing the stability and stiffness is necessary. A cogent mirror lightweight method is required to obtain the optimum lightweight configuration. Various optimum design and lightweight methods on large spaceborne optical mirrors are available in previous studies. Maser and Soosaar made a comparison of the relative maximum deflections under self-weight loading between a solid disk mirror and three kinds of lightweight mirrors including triangular, square, and hexagonal cell (or honeycomb) patterns using finite element analysis (FEA). The mirrors with a diameter of 12 inches were made of CerVit and had continuous simple support around the mirror edge. They concluded that the stiffness-to-weight ratios are well improved for the three kinds of lightweight mirrors.

\footnotetext{
* Corresponding author

E-mail:ethan@cc.ncu.edu.tw
}

In addition, the mass reduction effect is significant on the honeycomb pattern lightweight mirrors as all deflections are essentially kept equal (Maser and Soosaar 1972). An unconventional optimized large lightweight mirror with an elliptic shape was designed by Genberg and Cormany based on an automated nonlinear programming methodology. Faceplate thickness, core cell wall thickness, mirror overall thickness and effective cell spacing of the lightweight square core were adopted as the design variables. This optimized design was proven to be improved in diminishing deflections under loads and reducing mirror mass (Genberg and Cormany 1993). Park et al. (2005) explored a topology method to minimize the objective function of root-mean-square (RMS) surface error under self-weight loading and polishing pressure to obtain an optimum lightweight pattern. The lightweight mirror with a mass reduction ratio (MRR) of 0.65 was also compared with a classical lightweight mirror with honeycomb patterns to verify improvement under the horizontal position and polishing pressure load. They also manufactured the optical mirror blank based on the optimum lightweight pattern. Zhang and Yang (2006) used a genetic 
algorithm linked with FEA to design a lightweight mirror. An ideal lightweight configuration with the optimized variables was gained as the RMS optical mirror surface error under dead weight meets the given tolerance. Temperature control strategy on the lightweight design of a $\mathrm{SiO}_{2}$ mirror was used by Tan and Long (2010). The lightweight mirror had a surface temperature gradient less than $0.001 \mathrm{~K} \mathrm{~mm}^{-1}$ was achieved and the MRR was around 0.7.

The aforementioned analyses provided important information with regard to the optimum lightweight design for spaceborne mirrors. However, most of the previous studies are limited to self-weight loads and lack evaluations of each discrete lightweight geometrical parameter comprehensively. Moreover, some detailed structures such as processed flat surfaces or fillets on the mirror edge should be performed to obtain accurate results. A complete parametric opto-mechanical design and analysis during launch must be executed for the mirror lightweight. Hence, this study is devoted to using FEA and Zernike polynomial fitting under the quasi-static launch loads to obtain the optimum lightweight configuration for the primary mirror (M1) with open-back (Seibert 1990) hexagonal honeycomb cells based on the Taguchi Method (Byrne and Taguchi 1987). By selecting the control factors and levels for the honeycomb structures and other associated geometries appropriately, the whole optimization process was carried out based on the Taguchi Method with a proposed effective stiffness-to-weight ratio where the MRRs were divided by the product of the corresponding maximum mirror structural deflections and optical surface peak-to-valley (P-V) wavefront aberrations under the applied launching accelerations. The attained optimum lightweight spaceborne mirror will be verified numerically whether it passes through the space specification mechanical requirements. An optimum lightweight mirror blank made of ZERODUR ${ }^{\circledR}$ will be manufactured in practice.

\section{METHODOLOGY}

\subsection{Space Specification}

The present space-based RSI system must satisfy the following stiffness requirements: (1) The RSI system mass budget is $95 \mathrm{~kg}$. (2) The M1 (Fig. 1a) must exhibit a first natural frequency $\left(f_{n}\right)$ larger than $800 \mathrm{~Hz}$ in any direction on a rigid interface. (3) The M1 shall be designed to withstand a quasi-static load of $12 \mathrm{~g}$ (launching acceleration) at axial direction (X-axis) (Fig. 1a) and $6 \mathrm{~g}$ (vibration induced acceleration) at lateral direction (Z-axis) during launch. (4) The M1 structure load safety factor is specified as 1.5 against the ultimate limit.

\subsection{Key Mirror Dimensions and Configurations}

A Cassegrain-type telescope (Fig. 1b) was adopted in the present spaceborne RSI system including a M1, a sec- ondary mirror (M2) (Fig. 1a), and other truss and mechanical structures. The M1 is mounted using isostatic mounts (ISMs) as the key system component. The spaceborne RSI system development progress can be distinguished into system design and analysis phase, experimental model, structure model, and protoflight model (PFM). The PFM M1 has a diameter of $466 \mathrm{~mm}$, a central hole of $180 \mathrm{~mm}$ and a weight of $19.35 \mathrm{~kg}$ before light weighting, as shown in Fig. 2a. The PFM M1 has an aspheric surface (front surface) which will be coated with a silver layer to form an optical reflector and a flat surface (rear surface) perpendicular to the optical axis (Z-axis) which will be ground with hexagonal honeycomb cells. Two chamfers with radii of 2 and $5 \mathrm{~mm}$ are applied on the front and rear surface edges respectively to avoid glass chipping phenomena. Without high-order aspheric terms, the rotationally symmetric aspheric surface with the sag can be defined as:

$z=\frac{c \rho^{2}}{1+\sqrt{1-(1+k) c^{2} \rho^{2}}}$

where $c=-0.00051$ is the base curvature at the mirror surface vertex, $\rho$ denotes the radial coordinate measured perpendicular from the optical axis, and $k=-1.140251$ is the conic constant (hyperboloid mirror surface). The distance from the hyperboloid surface center to the rear surface is $45 \mathrm{~mm}$, indicating that the outermost mirror edge thickness is $58.13 \mathrm{~mm}$ (Fig. 2a). There are six fabricated flat surfaces of equal area of $43.13 \mathrm{~mm}^{2}$ arranged evenly with a $60^{\circ} \mathrm{in}-$ terval on the outermost edge (Fig. 2a) and three of the six surfaces in a $120^{\circ}$ interval are chosen as the attached ISM positions (Fig. 1a).

\subsection{Geometric Modelling and Lightweight Configurations for the Taguchi Method}

Removing material from the rear surface of the M1 reduces the mirror mass and eliminate the mass budget limit of the space-based RSI system. However, the mirror needs to maintain an adequate strength level to avoid optical performance decay during assembly on the ground or mirror destruction during launch. It is worth determining the optimum lightweight configuration for the M1 to minimize both the mirror mass and deflection under quasi-static launch loads. Therefore hexagonal honeycomb structures have been adopted as the lightweight configuration due to the highest stack density and the relatively high strength-to-weight ratio compared to other lightweight patterns (Maser and Soosaar 1972; Lin et al. 2010). The variations in honeycomb dimensions directly affect the mirror stiffness and MRR values. Four geometrical parameters including the faceplate thickness (A) (i.e., the depth of the honeycomb structure), the honeycomb structure rib thickness (B), the inscribed honeycomb structure circle diameter (C), and the inner/outer circle edge thickness (D) 
(i.e., the distribution range of the honeycomb cells) (Fig. 2b) are selected as control factors for the Taguchi Method. Furthermore, three adequate levels (Table 1) for each control factor are derived from the previous literatures (Park et al. 2005; SCHOTT AG 2008) and general mechanical process abilities. The total degrees of freedom in the analysis are hence $(3-1) \times 4=8$, and nine lightweight configurations are needed. According to the control factors and their levels (Table 1) and the orthogonal array of $\mathrm{L}_{9}\left(3^{4}\right)$ (Byrne and Taguchi 1987), the nine preliminary lightweight configurations numbered sequentially from Cases 1 to 9 are depicted in Fig. 3. For Case $1(\mathrm{~A}=9 \mathrm{~mm}, \mathrm{~B}=6 \mathrm{~mm}, \mathrm{C}=40 \mathrm{~mm}$, and $\mathrm{D}=$ 10/15), the M1 mass is reduced from 19.35 to $9.79 \mathrm{~kg}$ and the MRR is 0.49 . The MRRs of the other eight lightweight cases are between 0.45 and 0.57 as shown in Table 2. The FEA and Zernike polynomial fitting are then applied to the nine preliminary lightweight mirrors to obtain the 3D total deformation (TD), optical wavefront aberration and signal-to-noise ratio $(\mathrm{S} / \mathrm{N})$ results for the Taguchi analysis.

\subsection{Finite Element Modelling and Physical Properties}

The M1 is made of ZERODUR ${ }^{\circledR}$ glass ceramic whose coefficient of thermal expansion is close to zero (class 0 ), density is $2530 \mathrm{~kg} \mathrm{~m}^{-3}$, Young's modulus is $91 \mathrm{GPa}$ and Poisson's ratio is 0.24 (Döhring et al. 2007). The fracture criterion will be determined by the maximum principle stress (MPS) value of the mirror structure and the original surface conditions (Table 3) since ZERODUR ${ }^{\circledR}$ is a brittle material. As the induced highest MPS value is less than the ultimate strength (characteristic strength $\sigma_{0}$ ) divided by the safety factor (1.5), the mirror structure is considered safe. Computationally the static equilibrium equation with negligible inertia and damping effects is used to govern the deformation and stress-strain behavior of the assumed linear elastic mirror structure, and in the form of:

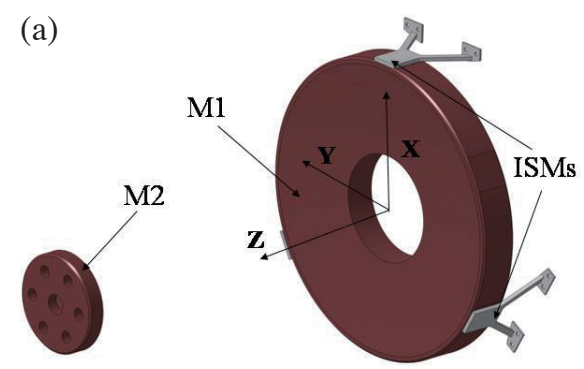

(b)

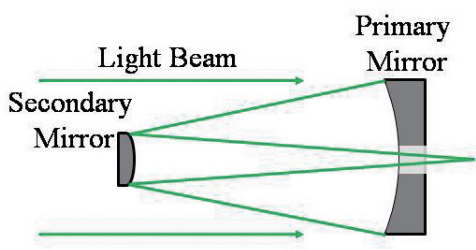

Fig. 1. (a) Schematic of the light path in a Cassegrain telescope; (b) Schematic of the simplified spaceborne RSI system. (Color online only)
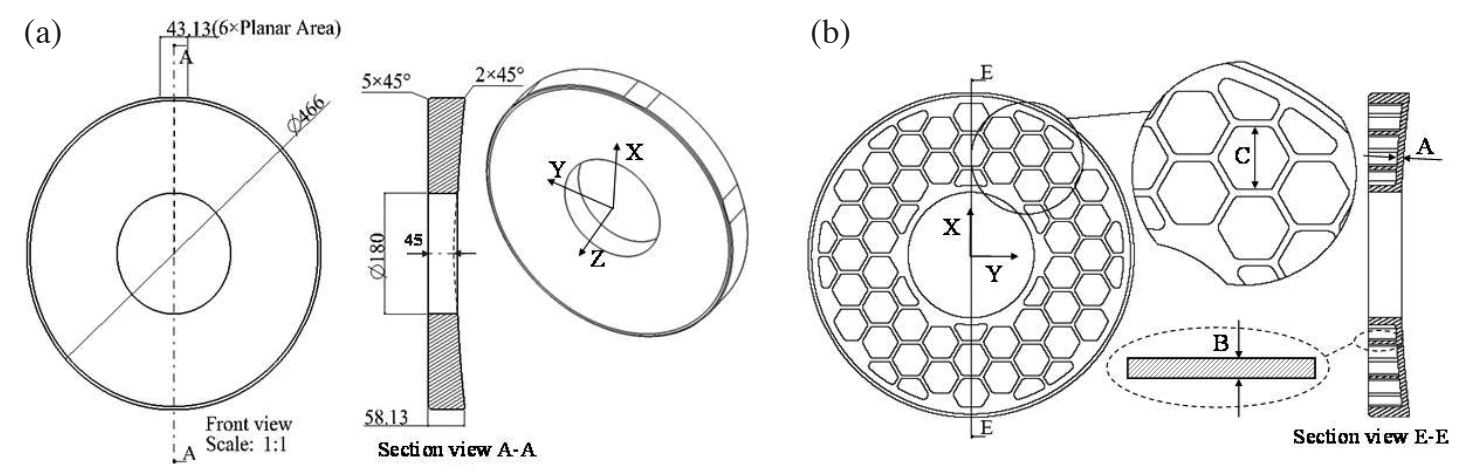

Fig. 2. (a) Schematic drawing of the PFM M1 (before lightweighting); (b) Schematic of the hexagonal honeycomb patterns on the rear surface.

Table 1. Control factors and their levels.

\begin{tabular}{ccccc}
\hline Factor & Description & Level 1 & Level 2 & Level 3 \\
\hline A & Faceplate thickness $(\mathrm{mm})$ & 9 & 12 & 15 \\
B & Rib thickness of the honeycomb structure $(\mathrm{mm})$ & 6 & 5 & 4 \\
C & Inscribed circle diameter of the honeycomb structure $(\mathrm{mm})$ & 40 & 45 & 50 \\
D & Inner / outer circle edge thickness $(\mathrm{mm} / \mathrm{mm})$ & $10 / 15$ & $10 / 10$ & $15 / 10$ \\
\hline
\end{tabular}




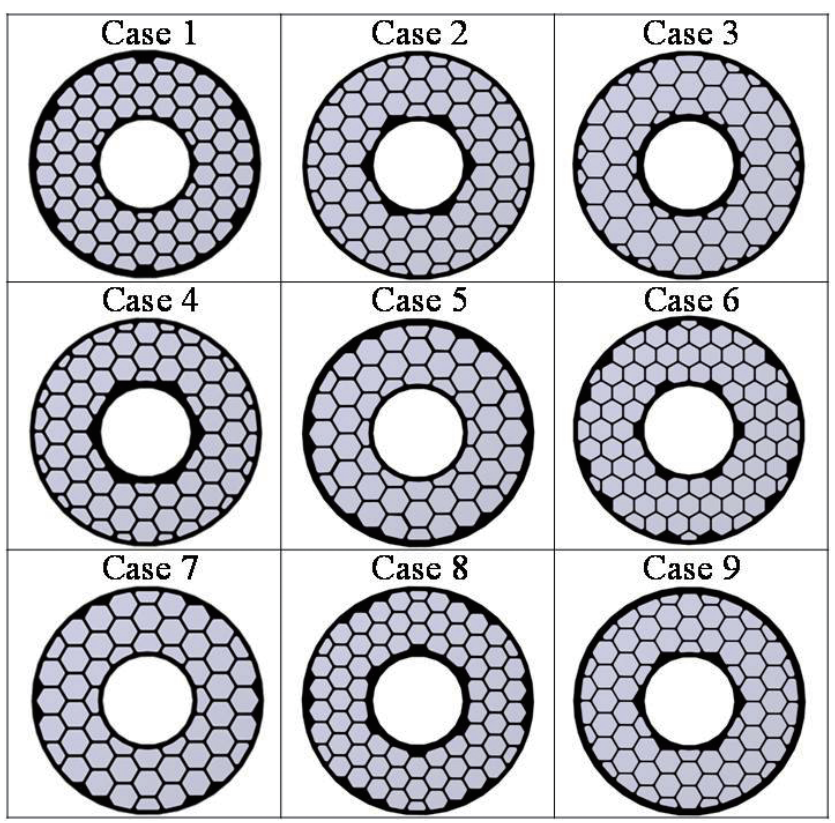

Fig. 3. Nine preliminary lightweight configurations in terms of the $\mathrm{L}_{9}\left(3^{4}\right)$ orthogonal array. (Color online only)

Table 2. MRR, $\mathrm{TD}_{\max }, \mathrm{P}-\mathrm{V}$ wavefront aberration, $\mathrm{y}$ and $\mathrm{S} / \mathrm{N}$ values for Cases 1 - 9 at the appled acceleration conditions.

\begin{tabular}{c|c|c|c|c|c|c|c}
\hline \multirow{2}{*}{ Case } & \multirow{2}{*}{ MRR } & \multicolumn{2}{|c|}{$\mathbf{1 2 g}$ in X-axis and 6g in Z-axis } & \multicolumn{2}{|c|}{$\mathbf{1 2 g}$ in X-axis and -6g in Z-axis } & \multirow{2}{*}{$\left(\mathbf{1} \mathbf{~ m}^{-2}\right)$} & \multirow{2}{*}{$\mathbf{S} / \mathbf{N}$} \\
\cline { 3 - 6 } & & $\mathbf{T D}_{\max }(\boldsymbol{\mu m})$ & $\mathbf{P - V}$ WA $(\boldsymbol{\mu m})$ & $\mathbf{T D}_{\max }(\boldsymbol{\mu m})$ & $\mathbf{P - V ~ W A}(\boldsymbol{\mu m})$ & & \\
\hline 1 & 0.49 & 1.39 & 1.25 & 1.51 & 1.24 & 0.136 & 102.8 \\
2 & 0.56 & 1.59 & 1.44 & 1.74 & 1.43 & 0.141 & 103.1 \\
3 & 0.57 & 1.72 & 1.53 & 1.81 & 1.57 & 0.135 & 102.7 \\
4 & 0.49 & 1.62 & 1.46 & 1.74 & 1.47 & 0.099 & 100.0 \\
5 & 0.50 & 1.53 & 1.38 & 1.62 & 1.41 & 0.114 & 101.3 \\
6 & 0.51 & 1.84 & 1.79 & 1.94 & 1.73 & 0.077 & 97.8 \\
7 & 0.48 & 1.88 & 1.75 & 1.99 & 1.72 & 0.071 & 97.3 \\
8 & 0.45 & 1.86 & 1.72 & 1.97 & 1.69 & 0.069 & 96.9 \\
9 & 0.47 & 1.47 & 1.37 & 1.55 & 1.36 & 0.114 & 101.3 \\
\hline
\end{tabular}

Table 3. $\mathrm{f}_{\mathrm{n}}$ and MPS $_{\max }$ values for Cases 1 - 9 at the appled acceleration conditions.

\begin{tabular}{c|c|c|c}
\hline \multirow{2}{*}{ Case } & \multirow{2}{*}{$\mathbf{f}_{\mathbf{n}}(\mathbf{H z})$} & $\mathbf{1 2 g}_{\mathbf{n}}$ in $\mathbf{X}$-axis and 6g in Z-axis & $\mathbf{1 2 g}$ in X-axis and -6g in Z-axis \\
\cline { 3 - 4 } & & $\mathbf{M P S}_{\max }(\mathbf{M P a})$ & $\mathbf{M P S}_{\max }(\mathbf{M P a})$ \\
\hline 1 & 1217.1 & 3.48 & 1.49 \\
2 & 1172.2 & 4.83 & 1.44 \\
3 & 1095.5 & 7.23 & 2.30 \\
4 & 1158.4 & 4.43 & 1.97 \\
5 & 1181.6 & 3.69 & 1.99 \\
6 & 1090.3 & 4.12 & 2.06 \\
7 & 1064.7 & 5.99 & 2.81 \\
8 & 1087.6 & 3.86 & 2.32 \\
9 & 1184.8 & 3.37 & 1.78 \\
\hline
\end{tabular}




$$
[K]\{u\}=\{F\}
$$

where $[K]$ is the stiffness matrix of the mirror structure, $\{u\}$ is the displacement vector, and $\{F\}$ is the applied force vector.

The corresponding unstructured grid system shown in Fig. 4 was constructed on the 3D computational domain for analyzing the PFM M1 structural results with FEA. The element type is Solid 186 and there are three degrees of freedom in each node. The three flat surfaces arranged evenly with a $120^{\circ}$ interval for the ISM attachment (Fig. 1a) are clamped $(u=0)$ and other mirror structures are free. Figure 5 shows that the whole M1 is subjected to the quasi-static loads of $12 \mathrm{~g}$ acceleration in the $\mathrm{X}$-axis (axial direction) and $\pm 6 \mathrm{~g}$ in the Z-axis (lateral direction). For simplification, the acceleration conditions will be divided into: (a) $12 \mathrm{~g}$ acceleration in the $\mathrm{X}$-axis and $6 \mathrm{~g}$ in the Z-axis, and (b) $12 \mathrm{~g}$ acceleration in the $\mathrm{X}$-axis and $-6 \mathrm{~g}$ in the $\mathrm{Z}$-axis in the present study. With the above well-posed boundary conditions (BCs), the finite element model consists of 136559 nodes determined by the convergence test. The results from the other eight lightweight cases are then calculated at the same mesh density. The undamped motion equation is used to govern the natural frequencies and mode shapes of the M1 structure and described as:

$[M]\{\ddot{u}\}+[K]\{u\}=0$

where $[M]$ is the mass matrix of the mirror structure and $\{\ddot{u}\}$ is the acceleration vector.

\subsection{Optical Wavefront Aberration Analysis}

The calculated deflections of the optical reflective surface can be transferred and fitted into Zernike polynomial coefficients and surface configurations with RMS and P-V wavefront aberrations. The Zernike polynomials are one of an infinite number of complete sets of polynomials in two orthogonal variables that are in continuous fashion within
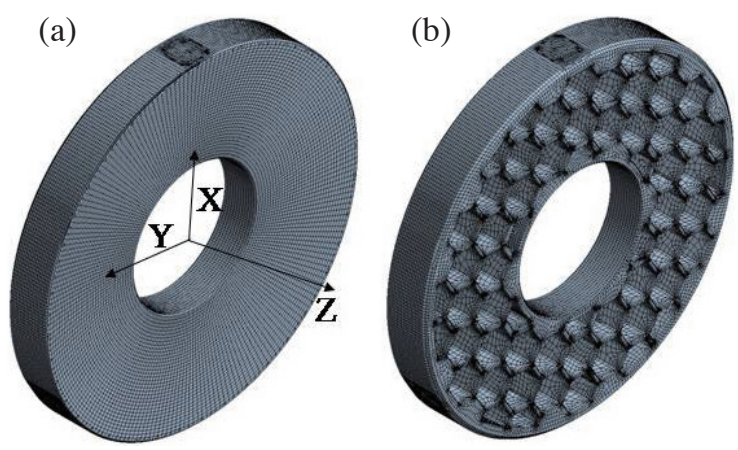

Fig. 4. Unstructured grid system for the PFM optical M1: (a) front side; (b) rear side. (Color online only) the interior of a unit circle. The Zernike polynomial series for wavefront aberrations can be written as:

$$
\begin{aligned}
\Delta Z(r, \theta)= & G_{00}+\sum_{n=2}^{\infty} G_{n 0} R_{n}^{0}(r)+ \\
& \sum_{n=1}^{\infty} \sum_{m=1}^{n} R_{n}^{m}\left[G_{n m} \cos (m \theta)+H_{n m} \sin (m \theta)\right]
\end{aligned}
$$

where $G$ and $H$ are individual Zernike polynomial coefficients, $R$ is the radial polynomials, $n$ and $m$ denote nonnegative integers $(n \geq m), r$ is the normalized radial distance $(0 \leq r \leq 1)$ and $\theta$ is the azimuthal angle.

\section{RESULTS AND DISCUSSION}

\subsection{D Structural Results}

Case 1 is investigated first and its TD and MPS distributions at $12 \mathrm{~g}$ acceleration in the $\mathrm{X}$-axis and $6 \mathrm{~g}$ in the $\mathrm{Z}$-axis are shown in Fig. 6. The TD distribution of the mirror is trilateral symmetry due to the limitation of the three clamped ISM positions and bends backward to the minus $\mathrm{Z}$-axis as a result of the quasi-static load of $6 \mathrm{~g}$ acceleration in the Z-axis. The maximum radial displacement of a calculated value of $1.39 \mu \mathrm{m}$ is located at the two mirror shoulders with red colored contours (Fig. 6a). The maximum MPS $\left(\mathrm{MPS}_{\max }\right)$ is found to be on the ISM positions with a calculated value of $3.48 \mathrm{MPa}$ (Fig. 6b) which is significantly lower than $10.35 \mathrm{MPa}$, the micro-yield strength of ZERODUR ${ }^{\circledR}$ with moderate surface treatment conditions (Yoder 2005; SCHOTT AG 2009).

As the lateral (Z-axis) acceleration is altered from $6 \mathrm{~g}$ to $-6 \mathrm{~g}$ and the axial (X-axis) acceleration remains $12 \mathrm{~g}$, the resulting TD and MPS distributions are shown in Fig. 7. The mirror bends forward to the positive Z-axis and has a maximum $\mathrm{TD}\left(\mathrm{TD}_{\max }\right)$ of $1.51 \mu \mathrm{m}$ at the mirror bottom with red colored contours (Fig. 7a). The MPS ${ }_{\max }$ is also on the ISM positions with a calculated value of $1.49 \mathrm{MPa}$ (Fig. 7b) and other MPS with higher values is concentrated on the lower portion of the mirror. The other eight lightweight

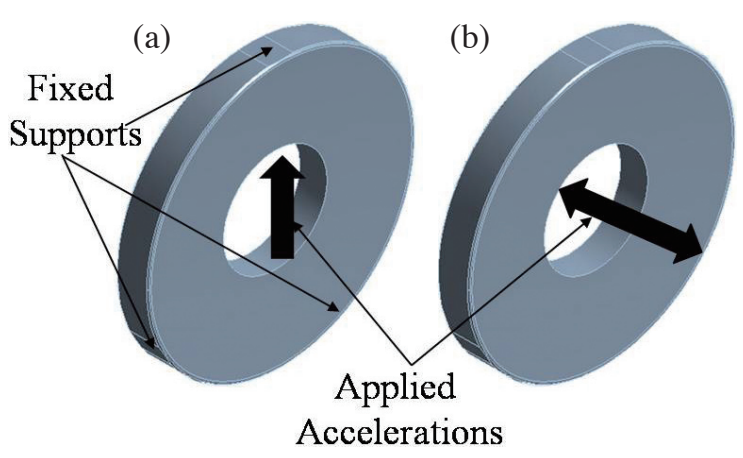

Fig. 5. Boundary conditions and applied accelerations: (a) $12 \mathrm{~g}$ in the $\mathrm{X}$-axis; (b) $6 \mathrm{~g}$ in the $\pm \mathrm{Z}$-axis. (Color online only) 
(a)

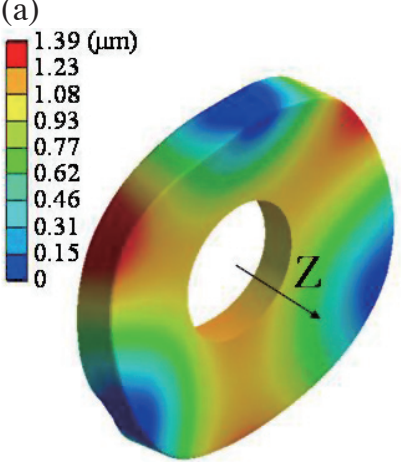

(b)

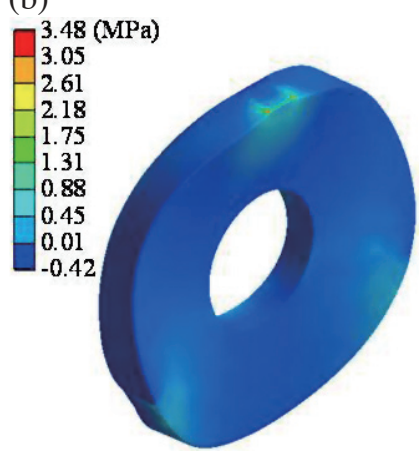

Fig. 6. (a) TD and (b) MPS distributions for Case 1 at $12 \mathrm{~g}$ acceleration in the $\mathrm{X}$-axis and $6 \mathrm{~g}$ in the $\mathrm{Z}$-axis. (Color online only)

cases have similar results with Case 1, while various control factors and levels leading to different $f_{n}$, MPS $_{\max }$, MRR and $\mathrm{TD}_{\max }$ values under the same BCs, which are summerized in Tables 2 and 3. Note that all $f_{n}$ values meet the space requirements $(800 \mathrm{~Hz})$ in Table 3 but the safety factor in Case 3 is higher than 1.5 at $12 \mathrm{~g}$ acceleration in the $\mathrm{X}$-axis and $6 \mathrm{~g}$ in the Z-axis, which motivates this study on the optimum lightweight M1 configuration.

\subsection{Zernike Polynomial Fitting Results}

The wavefront aberration distributions (Fig. 8) derived from the calculated structural deflections of the optical reflective surface are fitted by the Zernike polynomials with 66 terms. As the mirror is subject to the quasi-static loads of $12 \mathrm{~g}$ in the $\mathrm{X}$-axis and $6 \mathrm{~g}$ in the $\mathrm{Z}$-axis, the mirror surface deflects to the negative $\mathrm{Z}$-axis direction. Moreover, when the applied lateral acceleration is altered from $6 \mathrm{~g}$ to $-6 \mathrm{~g}$, the mirror surface deflects to the positive $\mathrm{Z}$-axis direction. Although the acceleration conditions are different, it is found that the two results consist of the same aberrations such as trefoil, focus, piston, and spherical aberrations due to the three constrained flat surfaces used for the ISM attachment. The P-V wavefront aberration values for Case 1 are also close $(1.25$ and $1.24 \mu \mathrm{m})$ at the two applied acceleration conditions, as shown in Table 2.

\subsection{Taguchi Analysis Results}

The purpose of this paper is to identify the optimum lightweight M1 configuration under the space specification requirements, i.e., to remove the mirror material for the mass reduction and maintain mirror stability and stiffness simultaneously. Some trade-offs have to be made when carrying out the optimization process. Therefore, the effective stiffness-to-weight ratio, the MRRs divided by the product of the RMS of the $\mathrm{TD}_{\max }$ values and the RMS of the P-V wavefront aberration values at the two acceleration condi- (a)

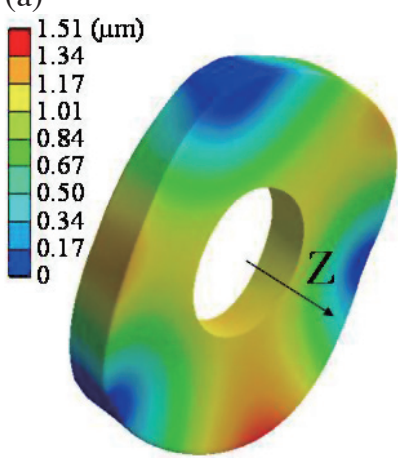

(b)

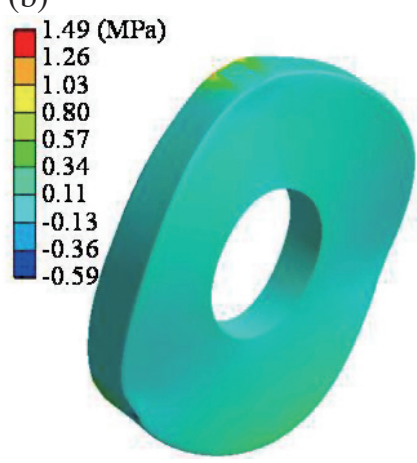

Fig. 7. (a) TD and (b) MPS distributions for Case 1 at $12 \mathrm{~g}$ acceleration in the $\mathrm{X}$-axis and -6g in the Z-axis. (Color online only)

tions, have been chosen as the optimum-evaluation parameter (y) in this study. Higher MRR, less $\mathrm{TD}_{\max }$, and less P-V wavefront aberration values resulting in a large y value are preferred for attaining the optimization target. Thus, the larger-the-better characteristic is adopted for the S/N estimations and is expressed as:

$\mathrm{S} / \mathrm{N}=-10 \log \left[\frac{1}{n} \sum_{i=1}^{n} \frac{1}{y_{i}^{2}}\right]$

where $n$ is the number of calculations. The MRR, TD ${ }_{\max }$, $\mathrm{P}-\mathrm{V}$ wavefront aberration, and corresponding y and $\mathrm{S} / \mathrm{N}$ values for the nine lightweight cases are listed in Table 2.

Figure 9 shows the main effects of the four control factors with three levels on $\mathrm{S} / \mathrm{N}$ in terms of the data set in Table 2. It is observed that factor A or the faceplate thickness is clearly the best choice for maximizing $\mathrm{S} / \mathrm{N}$ (robustness) for the largest slope of the curve of A versus $S / N$. The influences of $\mathrm{C}$ and $\mathrm{D}$ on $\mathrm{S} / \mathrm{N}$ are intermediate; on the other hand, factor B has only a slight weighting towards maximizing $\mathrm{S} / \mathrm{N}$. Therefore, the best settings for the control factor levels to maximize $\mathrm{S} / \mathrm{N}$ are $\mathrm{A} 1=9 \mathrm{~mm}, \mathrm{~B} 3=4 \mathrm{~mm}, \mathrm{C} 2=$ $45 \mathrm{~mm}$, and D1 = 10/15 (Table 1).

\subsection{Optimum and Feasible Parameters}

Since factor B (the rib thickness of the honeycomb structure) has a small influence (13\%) on S/N (Fig. 9) and the geometric parameters are independent on each other, the other two levels of B (B1 and B2) are also taken into account in the analysis. Thus, three preliminary optimum lightweight configurations, Cases a - c, are obtained and depicted in Fig. 10. The lightweight parameters and the corresponding MRRs and weights for Cases a - $\mathrm{c}$ are shown in Table 4, and the calculated $\mathrm{TD}_{\max }, \mathrm{P}-\mathrm{V}$ wavefront aberration, $\mathrm{y}$ and $\mathrm{S} / \mathrm{N}$ values for Cases a $-\mathrm{c}$ are listed in Table 5 . The lightweight configurations (Fig. 10) for Cases $a$ and $b$ are similar, and the MRRs (Table 4) for the two cases are 
considerably close. Since Case c exhibits the smallest rib thickness $(4 \mathrm{~mm})$ of the honeycomb structure, its mechanical process ability is easier without sacrificing the small cells around the inner and outer circle edges of the mirror and the MRR is up to 0.55 . Finally, Case $\mathrm{b}(\mathrm{A} 1=9 \mathrm{~mm}, \mathrm{~B} 3$ $=5 \mathrm{~mm}, \mathrm{C} 2=45 \mathrm{~mm}$, and D1 $=10 / 15$ ) has been chosen as the optimum lightweight pattern as a result of the highest $\mathrm{S} / \mathrm{N}$ (103.5) (Table 5).

Note that constant faceplate thickness (factor A) implies the bottom of the honeycomb structure is the same hyperboloid as the mirror reflective surface, which is not accessible with the general manufacutrability. Hence, effective depths for the honeycomb structures are adopted. As the lightweight pattern of Case b is hexad symmetry, the effective depths with a tolerence of $0.3 \mathrm{~mm}$ of the ten honeycomb cells (sequentially labeled in bold numbers in Fig. 11) are used, as shown in Table 6 . The MRR and $\mathrm{S} / \mathrm{N}$ values of Case $b$ with the effective depths of 0.5 and 103.5 respectively, proving the final optimum and feasible lightweight parameters are the effective depths (Table 6), $\mathrm{B}=5 \mathrm{~mm}, \mathrm{C}$ $=45 \mathrm{~mm}$, and $\mathrm{D}=10 / 15$. In addition, for Case $\mathrm{b}$ with the effective depths the calculated $\mathrm{f}_{\mathrm{n}}$ and MPS $\mathrm{S}_{\max }$ under the quasi- static loads of $12 \mathrm{~g}$ in the $\mathrm{X}$-axis and $6 \mathrm{~g}$ in the $\mathrm{Z}$-axis are $1252.1 \mathrm{~Hz}$ and $3.52 \mathrm{MPa}$, respectively, satisfying the space specification. Figure 11 shows the attained optimum lightweight PFM M1 with a mass of $9.72 \mathrm{~kg}$, and a ZERODUR ${ }^{\circledR}$ mirror blank based on the optimum design was achieved as well (Fig. 12). After bonding the three ISMs onto the M1 neutral plane, the calculated $f_{n}$ of the M1+ISM assembly is $440.3 \mathrm{~Hz}$ with the ISM bottom surfaces fixed (the specification for the M1 + ISM assembly is $300 \mathrm{~Hz}$ ). During integration and testing, the $\mathrm{M} 1$ is subjected to $1 \mathrm{~g}$ gravity in the $\mathrm{X}$-axis. The M1 surface has an RMS deformation of $5.7 \mathrm{~nm}$ and a P-V deformation of $34.8 \mathrm{~nm}$.

\section{CONCLUSION}

The FEA, Zernike polynomial fitting, and Taguchi Method were successfully performed to obtain the optimum lightweight configuration for a spaceborne M1 with honeycomb patterns for the PFM. The corresponding lightweight parameters of the honeycomb pattern are the derived effective depths, 5-mm rib thickness of the honeycomb structure, $45-\mathrm{mm}$ inscribed circle diameter of the honeycomb
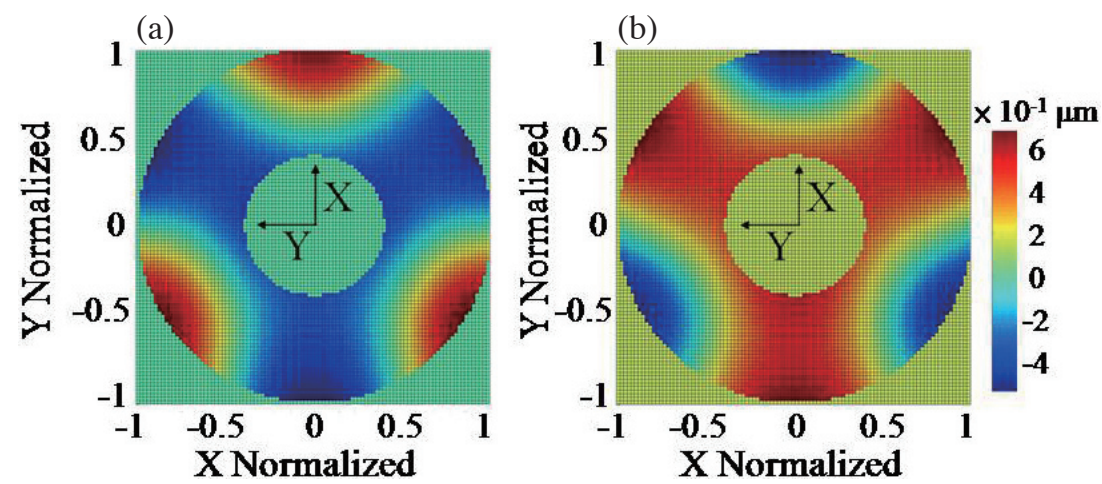

Fig. 8. Wavefront aberration distributions for Case 1 at (a) $12 \mathrm{~g}$ acceleration in the $\mathrm{X}$-axis and $6 \mathrm{~g}$ in the Z-axis and (b) $12 \mathrm{~g}$ acceleration in the $\mathrm{X}$-axis and $6 \mathrm{~g}$ in the $\mathrm{Z}$-axis. (Color online only)

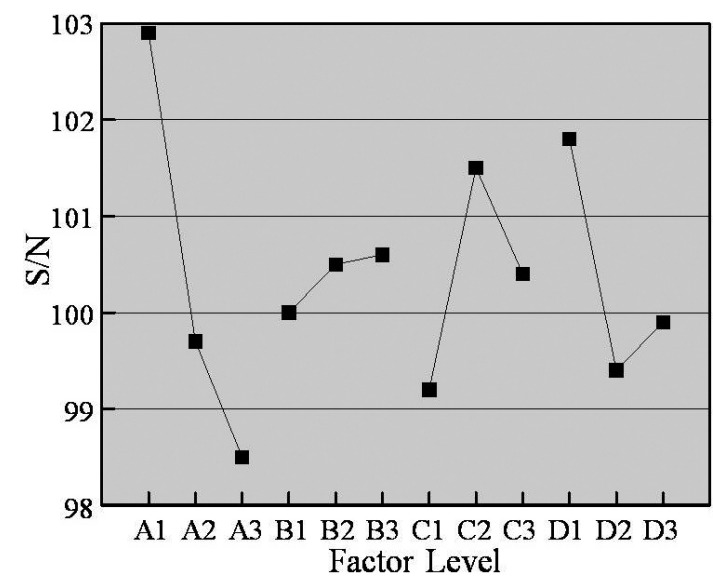

Fig. 9. Main effects plot on S/N. 


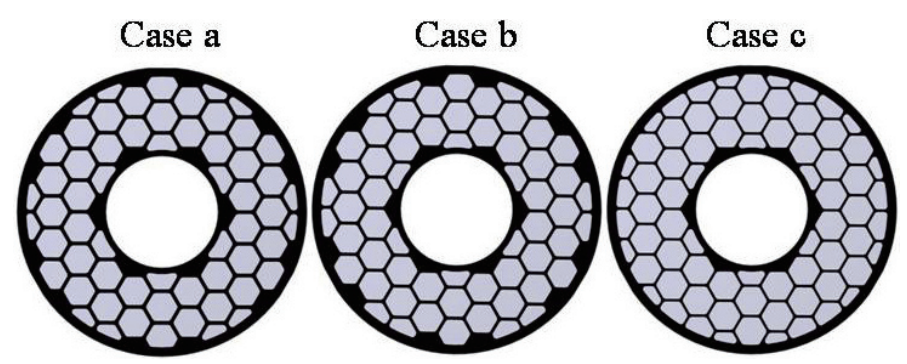

Fig. 10. Three preliminary optimum lightweight configurations. (Color online only)

Table 4. Lightweight parameters, MRRs and weights for Cases a - c.

\begin{tabular}{ccccccc}
\hline Case & $\mathbf{A}(\mathbf{m m})$ & $\mathbf{B}(\mathbf{m m})$ & $\mathbf{C}(\mathbf{m m})$ & $\mathbf{D}(\mathbf{m m})$ & MRR & Mirror Weight $(\mathbf{k g})$ \\
\hline a & 9 & 6 & 45 & $10 / 15$ & 0.48 & 10.01 \\
b & 9 & 5 & 45 & $10 / 15$ & 0.50 & 9.73 \\
c & 9 & 4 & 45 & $10 / 15$ & 0.55 & 8.75 \\
\hline
\end{tabular}

Table 5. $\mathrm{TD}_{\max }, \mathrm{P}-\mathrm{V}$ wavefront aberration, y and $\mathrm{S} / \mathrm{N}$ values for Cases a-c at the applied acceleration conditions.

\begin{tabular}{|c|c|c|c|c|c|c|c|}
\hline \multirow{2}{*}{ Case } & \multirow{2}{*}{ MRR } & \multicolumn{2}{|c|}{$12 \mathrm{~g}$ in $\mathrm{X}$-axis and $6 \mathrm{~g}$ in $\mathrm{Z}$-axis } & \multicolumn{2}{|c|}{$12 \mathrm{~g}$ in $\mathrm{X}$-axis and $6 \mathrm{~g}$ in - $\mathrm{Z}$-axis } & \multirow{2}{*}{$y\left(1 \mu \mathbf{m}^{-2}\right)$} & \multirow{2}{*}{$\mathbf{S} / \mathbf{N}$} \\
\hline & & $\mathbf{T D}_{\max }(\mu \mathrm{m})$ & P-V WA $(\mu \mathrm{m})$ & $\mathbf{T D}_{\max }(\mu \mathrm{m})$ & P-V WA $(\mu \mathrm{m})$ & & \\
\hline $\mathrm{a}$ & 0.48 & 1.34 & 1.20 & 1.43 & 1.19 & 0.145 & 103.4 \\
\hline $\mathrm{b}$ & 0.50 & 1.34 & 1.22 & 1.44 & 1.18 & 0.150 & 103.5 \\
\hline $\mathrm{c}$ & 0.55 & 1.42 & 1.31 & 1.51 & 1.26 & 0.146 & 103.3 \\
\hline
\end{tabular}

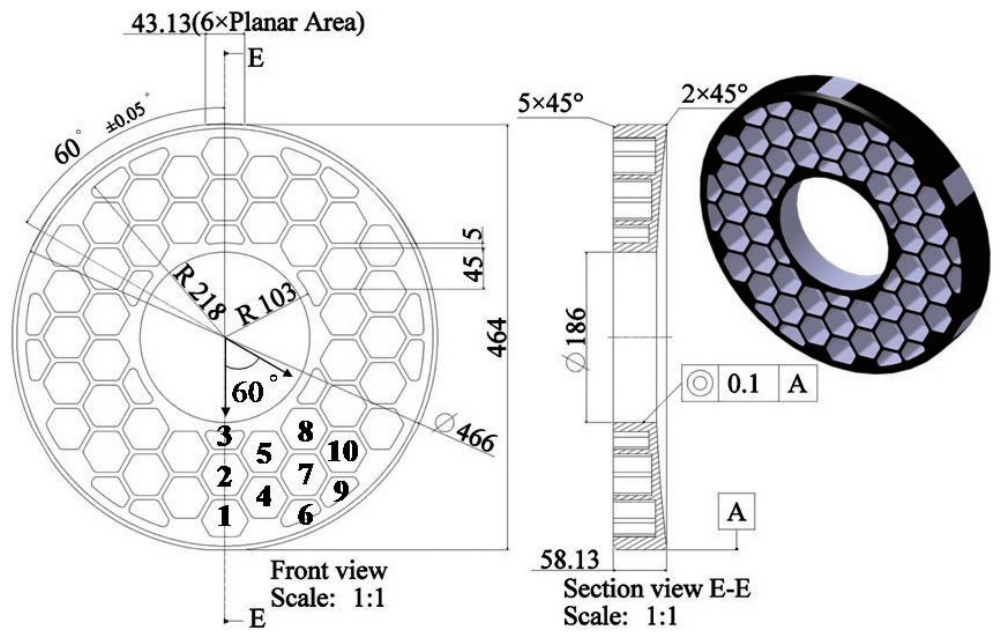

Fig. 11. Schematic drawing of the optimum PFM M1 (after light weighting). (Color online only)

Table 6. Effective depths of the honeycomb cells.

\begin{tabular}{ccccccccccc}
\hline NO. & $\mathbf{1}$ & $\mathbf{2}$ & $\mathbf{3}$ & $\mathbf{4}$ & $\mathbf{5}$ & $\mathbf{6}$ & $\mathbf{7}$ & $\mathbf{8}$ & $\mathbf{9}$ & $\mathbf{1 0}$ \\
\hline Depth (mm) & $46 \pm 0.3$ & $41 \pm 0.3$ & $39 \pm 0.3$ & $44 \pm 0.3$ & $41 \pm 0.3$ & $47 \pm 0.3$ & $44 \pm 0.3$ & $41 \pm 0.3$ & $47 \pm 0.3$ & $44 \pm 0.3$
\end{tabular}




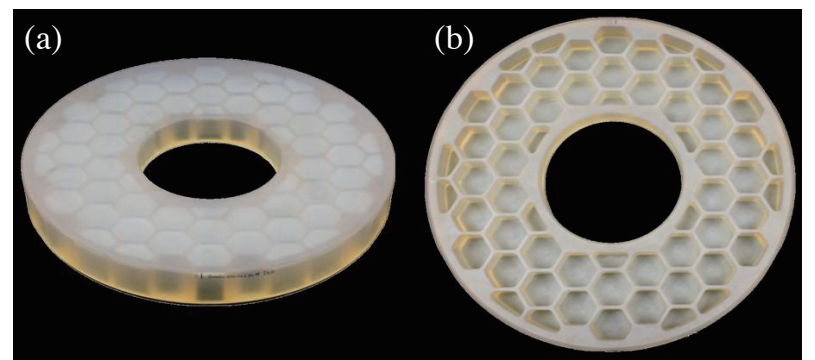

Fig. 12. Photographs of the optimum lightweight PFM M1 blank: (a) front view; (b) rear view. (Color online only)

structure, 10-mm inner circle edge thicknesses, and 15-mm outer circle edge thicknesses. The optimum M1 with a MRR of 0.5 and a mass of $9.72 \mathrm{~kg}$ was attained and examined to be approved by the system requirements and space specifications. The optimum lightweight mirror of ZERODUR ${ }^{\circledR}$ was manufactured.

Acknowledgements This research was mainly supported by the Ministry of Science and Technology (MOST), National Space Organization and Instrument Technology Research Center of National Applied Research Laboratories of Taiwan and also sponsored by the MOST Project under the grant number: MOST 104-2119-M-492-008.

\section{REFERENCES}

Byrne, D. M. and S. Taguchi, 1987: The Taguchi approach to parameter design. Qual. Progr., 20, 19-26.

Döhring, T., A. Thomas, R. Jedamzik, H. Kohlmann, and P. Hartmann, 2007: Manufacturing of lightweighted ZERODUR components at SCHOTT. Proc. SPIE, 6666, doi: 10.1117/12.733770. [Link]

Genberg, V. L. and N. Cormany, 1993: Optimum design of lightweight mirrors. Proc. SPIE, 1998, 60-71, doi: 10.1117/12.156631. [Link]

Lin, Y. C., L. J. Lee, and S. T. Chang, 2010: Integrated opto-mechanical analysis of optical mirror for remote sensing instrument. Instruments Today, 175, 80-93. (in Chinese)

Maser, K. R. and K. Soosaar, 1972: Elastic analysis of large spaceborne mirrors. Space Optics, Proc. of the $9^{\text {th }}$ International Congress of the International Commission for Optics, 184-207.

Park, K. S., J. H. Lee, and S. K. Youn, 2005: Lightweight mirror design method using topology optimization. Opt. Eng., 44, doi: 10.1117/1.1901685. [Link]

SCHOTT AG, 2008: TIE-38: Light weighting of ZERODUR $^{\circledR}$. SCHOTT Technical Information, TIE-38, SCHOTT North America.

SCHOTT AG, 2009: TIE-33: Design strength of optical glass and ZERODUR ${ }^{\circledR}$. SCHOTT Technical Information, TIE-33, SCHOTT North America, 10 pp.

Seibert, G. E., 1990: Design of lightweight mirrors. SPIE Short Course Notes.

Tan, F. and F. Long, 2010: Large mirror lightweight method based on temperature control strategy. Proc. SPIE, 7654, doi: 10.1117/12.867957. [Link]

Yoder, P. R., 2005: Opto-Mechanical Systems Design, Third Edition, CRC Press, 864 pp.

Zhang, W. and Y. Yang, 2006: Design of lightweight mirror based on genetic algorithm. Proc. SPIE, 6148, doi: 10.1117/12.674105. [Link] 